\title{
Rehabilitation in multiple sclerosis
}

\author{
Anna M. Kubsik-Gidlewska', A-E, Paulina Klimkiewicz ${ }^{1, B}$, Robert Klimkiewicz ${ }^{1, A}$, Katarzyna Janczewska ${ }^{1, C}$, Marta Woldańska-Okońska ${ }^{1, E, F}$ \\ Department of Rehabilitation and Physical Medicine, Division of Physiotherapy Military Medical Faculty, Medical University of Lodz, Poland \\ A - research concept and design; B - collection and/or assembly of data; C - data analysis and interpretation; \\ $D$ - writing the article; $E$ - critical revision of the article; $F$ - final approval of article
}

\section{Address for correspondence \\ Anna Kubsik-Gidlewska \\ E-mail:anna.kubsik@wp.pl \\ Funding sources \\ This work was supported by the \\ Medical University of Lodz research task \\ No. 502-03/5-139-04/502-54-098. \\ Conflict of interest \\ None declared}

Received on July 23, 2015

Revised on August 15, 2015

Accepted on March 22, 2016

DOI

\begin{abstract}
The aim of the study is to present a strategy of rehabilitation in multiple sclerosis on the basis of the latest developments in the field of physiotherapy. The publications on the problem discuss a wide range of methods of physiotherapy that can be used in order to reduce the degree of disability and alleviate the symptoms associated with the disease. The complexity of the disease, the difficulty in determining the appropriate treatment and a wide range of symptoms require a comprehensive approach to the patient, which would include both pharmacology and neurorehabilitation. Rehabilitation, which includes psychotherapy and symptomatic therapy, is regarded nowadays as the best form of treatment for multiple sclerosis. An indepth diagnostic assessment of functional status and prognosis should be carried out before the start of the rehabilitation process. The prognosis should take into account the mental state, the neurological status and the awareness of the patient. The kinesiotherapy program in multiple sclerosis is based on a gradation of physiotherapy which assumes a gradual transition from basic movements to more complex ones till global functions are obtained. The most appropriate form of treatment is functional rehabilitation combined with physical procedures. Recent reports indicate the need for aerobic training to be included in the rehabilitation program. The introduction of physical activities, regardless of the severity of the disease, will reduce the negative effects of akinesia, and thus increase the functional capabilities of all body systems.
\end{abstract}

Key words: physiotherapy, multiple sclerosis, physical activity, neurorehabilitation

\section{$10.17219 /$ acem/ 62329}

\section{Copyright}

Copyright by Author(s)

This is an article distributed under the terms of the

Creative Commons Attribution Non-Commercial License

(http://creativecommons.org/licenses/by-nc-nd/4.0/) 
Multiple sclerosis is a chronic inflammatory demyelinating disease of the central nervous system (CNS) the etiology of which is not fully understood and whose course is difficult to predict. The pathology and symptoms of the disease were first described by Jean-Martin Charcot in 1868. It is believed that a key role in the formation of demyelinating lesions may be played by autoimmune processes as well as environmental and genetic factors. The research into the pathophysiology of multiple sclerosis indicate that demyelinating changes affect the cerebral cortex and gray matter, including basal nuclei and cerebellar cortex. ${ }^{1}$ The process of inflammation, which is associated with the presence of pro-inflammatory cytokines and T-lymphocytes and macrophages, damages the blood - brain barrier, which is associated with neuronal malfunctioning and swelling, which in turn can can result in a relapse of the disease. ${ }^{2}$ Pathologists indicate that it may be oligodendrocytes, cells responsible for the construction of the myelin sheath around the axon, that cause the immune response. Damage to the myelin sheath and neurodegenerative processes lead to a reduction in the number of synapses and axons, and eventually to the loss of nerve cells. ${ }^{3}$ Viral infections, deficiency of vitamin D and the action of sex hormones are listed among many causes of the disease. Viral infections in combination with genetic factors may initiate an autoimmune process. An increasing rate of MS cases may also be associated with reduced exposure to the radiation of the sun. Insufficient exposure to sun radiation results in vitamin D deficiency, which reveals an immunoregulatory action preventing autoimmune inflammation of the brain and spinal cord. Sex hormones constitute an important factor influencing the development of MS, which is reflected in the increase of MS cases among women. Studies have shown that women suffering from MS who have higher levels of estradiol and low levels of progesterone experience a greater severity of the disease. ${ }^{4,5}$

Multiple sclerosis is a disease that affects young people. The occurrence of the disease in the world varies depending on latitude: the incidence increases with the rising latitude and the number of MS cases decreases nearer to the equator, which proves the correlation between MS and exposure to sun radiation. Currently, there are about 2.5 million people suffering from MS around the world and the numbers are growing. In Europe or the U.S. the incidence is $40-150$ patients per 100,000 inhabitants, while in Poland this figure is 55-57 per 100,000 inhabitants, which means that the country is at high risk of MS occurrence. ${ }^{6}$ The Caucasian race is the most vulnerable. The disease affects people of productive age leading to their disability. The greatest number of cases are observed between 20 and 40 years of age. MS is also diagnosed in children as childhood form of MS. People over 50 may be diagnosed with the late form of MS. ${ }^{7}$

\section{Rehabilitation in multiple sclerosis}

Multiple sclerosis affects most aspects of life of both the patients and their families. The complexity of the disease, the difficulty in determining the appropriate treatment and a wide range of symptoms call for a comprehensive approach to the patient, which would involve both pharmacology and neurorehabilitation. Physiotherapy, which is an integral part of rehabilitation, consists in kinesitherapy, physical therapy, massage and hydrotherapy. Physiotherapy in multiple sclerosis is aimed at improving mobility through compensation mechanisms that involve the activation of capabilities of effectors and behavior, which results in the patient regaining functioning, not movement. All applications included in physiotherapy should be recommended in such a way as to act upon the largest possible number of motor deficits. The introduction of physical activity, regardless of the severity of the disease, will reduce the negative effects of akinesia, and thus increase the functional capabilities of all body systems. ${ }^{8}$

In the period of acute illness, it is necessary to avoid immobilization of patients due to the consequences of akinesia. Physiotherapy of these patients involves frequent changes of body position in order to prevent bed sores, prevention of contractures using passive exercises, and the implementing breathing exercises to prevent complications of the respiratory system. What is essential in patient care is the prevention of infections of the urogenital system and assistance in activities of daily living.

The rehabilitation program should take into consideration the phase of the disease, degree of disability and neurological deficits. The therapy at the time of remission is therefore based on the degree of impairment and the objectives of treatment. The rehabilitation in this phase of the disease is conducted in hospitals, outpatients and the home, depending on the functional status of the patient. Before the implementation of physiotherapy, an in-depth diagnostic assessment of the functional status and prognosis should be carried out. The prognosis should take into account the mental state, the neurological status and the state of cognitive functions of the patient. ${ }^{9}$ The rehabilitation program is often hampered by fatigue associated with the disease, which limits not only the possibility of movement of the patient but also their mental functions. Improving motor skills of MS patients aims at enhancing muscle strength, normalizing muscle tension, improving coordination and balance, preventing urinary incontinence, increasing or maintaining the range of motion in the joints, preventing muscle atrophy and counteracting the consequences of immobilization. The continuity of the rehabilitation process should involve exercises that would eliminate the problems resulting from the disease. Therefore, daily rehabilitation would consist of balance and coordination exercises as well as breathing, stretching and relaxation 
exercises. It would also include aerobic training, unloaded exercises, exercises which strengthen particular groups of muscles, and improve proprioception. The rate of exercise and load should be such as not to cause fatigue and overheating of the body, which could result in increased muscle tension.

\section{Impairment-based approach}

Kinesis program is based on a pyramid of physiotherapy planning in multiple sclerosis, which assumes a gradual transition from basic movements to more complex ones until global functions are reached. The most appropriate form of therapy, that is functional rehabilitation, aims at the recovery of lost functions by performing movements in three dimensions. ${ }^{10}$ The methods designed to recreate natural human movement include Proprioceptive Nuromuscular Facilitation (PNF) and Bobath Neurodevelopmental Treatment (NDT). These neurophysiological methods allow the recovery of impaired functions of the body by using appropriate patterns of movement and techniques of coordination, stabilization and relaxation. It has been observed that the movements performed in everyday life are diagonal and multiplane. Therefore, patterns of movement for the upper limb, lower limb, neck and head have been developed. One of the techniques that both the methods employ is a manual technique of facilitation of movement (PNF). The purpose of this technique is to assist the patient to correctly perform a movement in a particular activity, thus preventing a pathological position of the body and assisting in the initiation and finishing of movement. ${ }^{11}$ The PNF method also includes gait re-education and exercises of facial muscles. It eliminates dysfunction of chewing, swallowing and breathing. Bobath neurodevelopmental concept assumes that the essence of motor deficits resulting from impairment in the CNS is a dysfunction in posture reflexes that are needed to coordinate movements in space. The correct muscle tension and active movements can be acquired by inhibiting the pathological patterns of posture. The advantage of these methods is the ability to work in various positions, including lying position on the mattress, which allows for the adjustment of the method of rehabilitation to the functional status of the patient. The use of these methods and their efficiency of the therapy has been confirmed in evidence based medicine (EBM). ${ }^{12}$

Problems with balance and coordination are common symptoms of the disease that cause difficulty in movement, so the inclusion of balance and coordination exercises into the therapy is necessary. These exercises increase stability during gait, prevent falls and enhance posture control. Coordination exercises consist of motor acts of specified complexity, which improve coherence of movement and reduce energy consumption which is due to deficiency of coordination. Coordination and balance exercises are carried out according to the Frenkel method, in which footprints painted on the floor are used to learn proper gait. The exercises are performed in 3 phases (right foot steps forward, shift weight, left foot joins the right one). Learning to sit down and stand up is part of this method. The movement is divided into three phases (feet withdrawn, forward leaning of the trunk, straightening of the legs and getting up). Balance and proprioceptive exercises in patients with stability in standing position are carried out with the use of stabilometric and posturometric platforms based on the biofeedback method. The examinations conducted with these devices prove better control of the posture in patients with multiple sclerosis. ${ }^{13}$ Coordination and balance exercises can be combined with a proprioception exercises that can be performed in sitting or standing positions. Proprioreception disorders are common symptoms of multiple sclerosis. They influence various stages of rehabilitation. The implementation of neuromuscular control training in the early stages of rehabilitation reduces the risk of further injuries resulting from loss of balance. ${ }^{14}$ Hippotherapy is an alternative form of exercise which improves balance and stability in patients with MS. Better balance and gait were observed in patients undergoing hippotherapy when compared to the control group in which classical physiotherapy was used. ${ }^{15}$

One of the many symptoms related to MS is spasticity, which often makes rehabilitation and care difficult and most importantly deepens the patient disability. Spastic paresis affects both lower and upper limbs with greater severity of pathological tension in the lower extremities. It occurs in $40-60 \%$ of patients with multiple sclerosis. It is a symptom of the impairment of upper motor neuron which reveals excessive activity of alpha cells of the anterior horn of the spinal cord. Mild spasticity is a beneficial phenomenon as it improves blood circulation in the limbs and prevents muscular atrophy. High degree of spasticity (3 or 4 in Ashworth scale) causes contractures, joint deformities and bedsores which lead to infections. Apart from pharmacotherapy, physiotherapy plays an important role in the cases of severe spasticity. The plan of the rehabilitation program should take into account the fact that the use of physical applications before kinesiotherapy has positive effects while greater physical efforts can increase muscle tension. ${ }^{16}$ The physical application which is used before kinesiotherapy is cooling the defined areas of the body, and even the spinal cord is treated with low temperatures. This is to produce the effect of local anesthesia in peripheral sensory receptors. The use of cold reduces responses to active stretching. Passive stretching is a contraindication, as it reduces the excitability of the motor neurons and maintains elasticity properties of muscles and joints. Cryotherapy is applied in the form of an ice-slush bath, ice massage, coldpacks (bags with frozen silicone gel), cryogenic suits and compression cryocuffs. Cryotherapy is also applied systemically in patients with MS to obtain an analgesic effect as well as reduce fatigue syndrome. ${ }^{17}$ 
Other physical methods used in the treatment of spasticity are electrotherapy and magnetic therapy. Electrical stimulation of neuromuscular system (NMES), transcutaneous electrical nerve stimulation (TENS), Hufschmidt method and functional electrical stimulation (FES) are listed among the applications of electrotherapy. Electrical stimulation seems to be the best method because damage to Upper Motor Neuron does not alter the excitability of the muscles to electrical stimuli, so that the impaired bioelectrical function of muscles can be replaced. The treatment parameters are determined on the basis of EMG or chosen according to the generally accepted standards. Electrical stimulation is applied with implant electrodes and methods of transcutaneous stimulation. Both methods result in a reduction of muscle tension, which leads to a wider range of motion in the joints and improved gait efficiency. The use of implant electrodes runs the risk of complications such as displacement of electrodes or the occurrence of adverse reaction of the body to the implant. ${ }^{18}$ Transcutaneous electrical nerve stimulation (TENS) is a method that has been used in the treatment of pain in patients with MS. Electrodes are placed in areas of pain or along the nerves. A segmental arrangement, on a specific dermatome, is also in use. The frequency ranges from 1 to $100 \mathrm{~Hz}$, and the value of intensity is determined for the patients according their individual feelings. Hufschmidt method is also used to normalize muscle tension through the stimulation of spastic muscles and their antagonists. Double square-wave impulses are used to achieve a functional balance between agonists and antagonists. This method allows for proprioceptive facilitation and affects alpha motorneurons. ${ }^{19}$ Functional electrical stimulation (FES) employs ministimulators that are applied to paretic muscles beyond the control of the central nervous system. The most frequent uses of FES treat the peroneal nerve in patients with hemiparesis in order to improve the various phases of gait. The electrical stimulation is carried out with rectangular pulse current with a frequency of $20-50 \mathrm{~Hz}$ and an impulse duration of $0.1-$ $0.2 \mathrm{~ms}$. Magnetic fields of low and high frequencies are also applied in patients with multiple sclerosis. Since the introduction of alternating magnetic fields into physiotherapy, the term magnetic therapy has referred to the action of magnetic field with induction values above $100 \mu \mathrm{T}$. The application of fields with induction $100 \mu \mathrm{T}$ is referred to as magnetic stimulation. The advantage of the application of magnetic fields is the fact that they are painless, athermal and permeable through the patient's tissue. In magnetotherapy, the frequency ranges from $2 \mathrm{~Hz}$ to 60 $\mathrm{Hz}$, and the induction can be up to $20 \mathrm{mT}$. The applied impulses can have a triangular, rectangular, sinusoidal and trapezoidal shapes. ${ }^{20}$ This application in patients with MS is used to control pain and decrease muscle tension. In the case of spasticity, the intermittent mission of magnetic fields is used starting with a $3 \mathrm{~s}$ break and then in each subsequent application the break is gradually shortened by $0.5 \mathrm{~s}$ till it reaches continuous emission. The analgesic effect results from the secretion of endogenous opiates from the group of $\beta$-endorphins, from the hyperpolarization of nerve endings, and also from a mechanism for reducing the permeability of cell membranes. ${ }^{21}$ Although magnetostimulation has only recently started to be used in treatment, it is widely used in MS patients, as has been indicated in numerous publications. The induction value in magnetostimulation does not exceed 100 $\mu \mathrm{T}$, the carrier frequency is about $3000 \mathrm{~Hz}$, and the basic frequency amounts to several $\mathrm{Hz}$. The general effects of magnetostimulation include the stimulation of life processes and the maintainance of homeostasis. In multiple sclerosis therapy, it is used in the form of systemic magnetostimulation applicator used as a mat. This results in the reduction of muscular tension, relaxation, which leads to the reduction of fatigue syndrome. ${ }^{22}$ The studies of magnetostimulation have confirmed the efficacy of this treatment for the secretion of hormones such as melatonin is related to an analgesic effect and serotonin the increase of which improves well-being and mood. ${ }^{23}$ Transcranial stimulation is used in order to stimulate the selected areas of the cerebral cortex. This process is used primarily in the treatment of retrobulbar optic neuritis in order to improve the conductivity of the optic tract. The application of reptitive transcranial magnetic stimulation (rTMS) in patients with MS resulted in a reduction of spasticity in the lower limbs and improvement of the neurogenic bladder dysfunction. ${ }^{24}$

MS patients also undergo therapy with the use of laser light. Due to the fact that laser treatment produced beneficial effects in other diseases, it has been included in the rehabilitation program for MS patients. Laser radiation of low and medium power induces, first of all, changes at the cellular level and furthermore demonstrates analgesic, anti-inflammatory and anti-oedematous actions. Laser biostimulation causes the creation of biologically active compounds and, as a result, leads to changes in cell metabolism. The regenerative effect of laser radiation is important for patients with MS due to the fact that it enhances the regeneration of demyelination in the CNS, which reduces nerve conduction. Biostimulating laser light affects the regeneration of nerves by stimulating the growth of Schwann cells enhancing nerve conduction. Helium-neon lasers of $10 \mathrm{~mW}$ power and $632 \mathrm{~nm}$ wavelength are used in the therapy in the form of "contact point" method. The area of the paraspinal nerve trunks along the line in the $\mathrm{C}-\mathrm{Th}$ and $\mathrm{Th}-\mathrm{L}$ regions are irradiated. ${ }^{25}$ Cold lasers, where cold is applied in combination with laser radiation, are also used as they decrease muscle tension and produce an analgesic effect. ${ }^{26}$

The rehabilitation program should also include the pelvic diaphragm muscle exercises due to the neurogenic bladder dysfunction, which occurs in $78 \%$ of patients with MS. Sphincter disorders occurring in the form of urinary incontinence or retention cause discomfort for patients 
and limit their participation in activities of daily living and social activities. Kegel isometric exercises, which involve tensing and relaxation of certain groups of muscles, are performed to exercise the pelvic diaphhragm, which results in an increase in muscle mass and strengthening and the growth of the resting tension of these muscles. The exercises allow for a prolonged contraction of the muscles, improving control of neuro-muscular and connective tissue to stabilize the pelvis, which, in turn, improves the mechanism of urination. Pelvic diaphragm muscle training should be combined with enhancement of deep muscles such as transversus abdominis mainly due to the synergistic action of these structures. Co-activation of these muscles is reduced or abolished in people with urinary incontinence. ${ }^{27}$ In addition, the training should also include gluteal muscles, the adductor muscle and the ischiocrural muscle group. The simultaneous use of Kegel exercises, EMG biofeedback and electrical stimulation (NMES) produces very good results. ${ }^{28}$

The neurological diseases, including MS, also cause respiratory disorders, which include difficulties in respiration due to inspiratory muscle weakness. Patients with dysphagia are at risk of aspiration of fluids resulting from the weakening of the muscles of the upper respiratory tract. The introduction of breathing exercises in the early stages of multiple sclerosis may prevent further complications. During the relapse, the patient usually remains in bed; therefore, breathing exercises often become the only form of activation. Breathing exercises are also used in patients with impaired speech in the form of speech therapy. ${ }^{29}$

In patients with multiple sclerosis, impaired gait is a consequence of neurological deficits such as balance and coordination disorders, spasticity, visual disturbances, paraparesis and hemiparesis. Deteriorated locomotion or movement in the form of pathological gait significantly reduces the quality of life of patients with MS and makes them dependent on family or friends. It is, therefore, necessary to include re-education of gait in the rehabilitation program. The re-education of gait should be preceded by a thorough analysis, which would aim to assess the disorder in different phases of gait compared to the normal gait pattern. One of the methods of re-education is learning to walk on a treadmill with body weight relief. This allows for the improvement of gait parameters and increases strength and endurance of the muscles of the lower limbs. LOKOMAT is a more advanced device which consists of a treadmill relief system and a computer control system of the movement of the lower limb orthoses connected to sensors. ${ }^{30}$

Another stimulus in the field of physiotherapy, which comprehensively acts on the body of the patient, is hydrotherapy. The physical properties of water such as hydrostatic pressure, hydrodynamic pressure, viscosity and buoyancy make the unloaded gymnastics of the motor system feasible so that it is possible to obtain a greater range of joint movement without pain components. Hy- drotherapy reduces the sensitivity of muscle fibers and skin receptors by reducing the activity of gamma neurons, which is manifested by a decrease in spasticity. The temperature of water plays the key role for patients with multiple sclerosis due to the possibility of the occurrence of Uhthoff's symptom; therefore, hot baths should be avoided. Thus, complete and partial baths at a temperature of $34-36^{\circ} \mathrm{C}$ are applied. The water at this temperature inhibits the flow of afferent impulses from proprioceptors to the CNS, which has and analgesic and relaxing action. Studies in patients with MS show that hydrotherapy greatly improves independence and mobility and reduces the symptoms of depression and fatigue. ${ }^{31}$

\section{Task-oriented approach}

Physical activity, which the World Health Organization recommends, is an essential part of maintaining the efficiency of the cardio-respiratory system and general health. The WHO recommends performing any physical activity of moderate intensity for $30 \mathrm{~min}$ a day, 5 days a week or 20 min a day for 3 days a week with high intensity. Regular physical activity in the form of sport or recreation results in significant health benefits. The introduction of physical exercise into the plan of physiotherapy for patients with multiple sclerosis have until recently aroused controversy, as there was a belief that physical effort may initiate a relapse of the disease. However, some studies published in recent years have refuted these arguments. On the contrary, they confirmed the efficacy of aerobic training in MS patients. The introduction of fitness training for patients with MS is conditioned by the requirement to maintain strict selection criteria for physical effort, which guaranties an increase in efficiency without exposing the patients to the possibility of progression of the disease. The training should take several weeks or even months. Most frequently a treadmill or a cycloergometer are used in this type of exercise. ${ }^{9}$

Physical activity in patients with multiple sclerosis improves not only their physical capacity, but also their mood and attitude towards the exercises. Better cardiorespiratory efficiency has been proven to enhance the cerebrovascular function, which suggests that movement can also significantly improve the brain function in patients with multiple sclerosis. ${ }^{32}$ Aerobic training increases muscle strength of the lower limbs, which manifests itself in reducing spasticity without the risk of relapse or the symptoms of fatigue. ${ }^{33}$

Recent recommendations for patients with multiple sclerosis suggest performing physical activities 2-3 times per week at an intensity of $50-70 \%$ of VO2max corresponding to $60-80 \%$ of maximum heart rate. The duration of the exercise should not exceed the initial period of $40 \mathrm{~min}$. The intensity should increase gradually depending on the degree of disability. The training should also 
include alternating resistance exercises at an 24-48 h interval. Progressive resistance training induces a compensatory growth of muscle fibers. It is recommended that fitness exercises should be performed by patients with the EDSS result of less than $7 .^{34}$

The presence of chronic fatigue syndrome, the Uhthoff symptom and pain may limit the rehabilitation program in the scope of physical activity. Studies indicate that patients who do not perform a physical activity have a significantly reduced quality of life and a greater degree of disability in comparison with the group that practices regularly. ${ }^{35}$

\section{Discussion}

The aforementioned literature points to the fact that multiple sclerosis patients require a wide range of rehabilitation proceedings. It also demonstrates difficulty in developing a personal rehabilitation program by therapists and doctors. Many researchers have noted the scale of the problem, which manifests itself in numerous scientific publications, conferences and symposiums dedicated to the topic. In his work, Wiles provides a summary of research presenting the standards of treatment in MS and an assessment of physiotherapeutic methods. ${ }^{36}$ There is a correlation between the degree of disability and the quality of life of patients. Rość proved that the quality of life depends on the degree of physical disability assessed with the Kurtzke Expanded Disability Status Scale (EDSS). ${ }^{37}$ In his research, Brochet presents a decrease in quality of life due to the pain that affects daily living. ${ }^{38}$ Numerous studies indicate that MS has a negative impact on physical and mental functioning of patients. The aim of doctors and physiotherapists in their work is the implementation of new methods of therapy to alleviate symptoms and, consequently, improve the quality of life.

Over the last few years, research has demonstrated a significant difference in the functional status between MS patients who undergo rehabilitation and those who are not rehabilitated. The inpatient rehabilitation has been proven to be more efficient than the outpatient counterpart. The current algorithm is based on symptoms and tasks. The symptom-based therapy is oriented towards reducing disability through targeting current neurological deficits. Functional rehabilitation and physiotherapy play an important role here. In contrast, the task-based therapy involves the introduction of physical effort, which has not been recommended so far, into the rehabilitation program. The role of daily physical activity, which is related to the functional status of the patient, has been underlined as well as the impact of physical inactivity on the reduction of the cardiopulmonary fitness level. ${ }^{39}$ Recent reports indicate that physical activity of patients with multiple sclerosis can be increased by providing them with some behavioral interventions. ${ }^{40}$

\section{References}

1. Vercellino $M$, Masera S, Lorenzatti $M$, et al. Demyelination, inflammation, and neurodegeneration in multiple sclerosis deep gray matter. J Neuropathol Exp Neurol. 2009;68(5):489-502.

2. Stadelmann C, Albert M, Wegner C, Bruck W. Cortical pathology in multiple sclerosis. Curr Opin Neurol. 2008;21:229-234.

3. Filippi M, Bozzali M, Rovaris M, et al. Evidence for widespread axonal damage at the earliest clinical stage of multiple sclerosis. Brain. 2003;126:433-437.

4. Munger $\mathrm{KL}$, Zhang SM, O'Reilly $\mathrm{E}$, et al. Vitamin $\mathrm{D}$ intake and incidence of multiple sclerosis. Neurology. 2004;62:60-65.

5. Bansil S, Lee HJ, Jindal S, Holtz CR, Cook SD. Correlation between sex hormones and magnetic resonance imaging lesions in multiple sclerosis. Acta Neurol Scand. 1999;99:91-94.

6. Kułakowska A, Bartosik-Psujek H, Hożejowski R, Mitosek-Szewczyk K, Drozdowski W, Stelmasiak Z. Selected aspects of the epidemiology of multiple sclerosis in Poland - a multicentre pilot study. Neurologia i Neurochirurgia Polska. 2010;44:443-452.

7. Selmaj K. Multiple Sclerosis - diagnostic criteria, the natural course of the disease. Pol Prz Neurol. 2005;1(3):99-105.

8. Kwolek A, Podgórska J, Rykała J. Own experience in the rehabilitation of multiple sclerosis patients. Przegląd Medyczny Uniwersytetu Rzeszowskiego. 2010;2:213-220.

9. Opara J. Physiotherapy in multiple sclerosis. Wydawnictwo AW, Katowice. 2012:16-70.

10. Woszczak M. Physiotherapeutic procedures in multiple sclerosis. Polski Przegląd Neurologiczny. 2005;1:130-133 (in Polish).

11. Klimkiewicz P, Kubsik A, Woldańska-Okońska M. NDT-Bobath method used in the rehabilitation of patients with a history of ischemic stroke. Wiad Lek. 2012;65:102-107.

12. Łada A. Diagnostics and neurological therapy in the light of the NDT- Bobath concept. Prakt Fizjo Rehab. 2010;4:30-34.

13. Kielnar R, Mraz M, Mraz M, Oleksy $Ł$, Dębiec-Bąk A, Chantsoulis M. Evaluation of postural stability based on a front and back stability margin of people with multiple sclerosis after rehabilitation. Acta Bio - Optica et Informatica Medica. Inzynieria Biomedyczna. 2009;15: 226-229.

14. Cattaneo D, Jonsdottir J, Zocchi M, Regola A. Effects of balance exercises on people with multiple sclerosis: A pilot study. Clin Rehabil. 2007;21(9):771-781.

15. Munoz-Lasa S, Ferriero G, Valero R, Gomez-Muniz F, Rabini A, Varela E. Effect of therapeutic horseback riding on balance and gait of people with multiple sclerosis. G Ital Med Lav Ergon. 2011;33:462-467.

16. Steiborn B, Łuczak-Piechowiak A. Kinesytherapeutic methods in the treatment of spasticity. Polski Przegląd Nauk o Zdrowiu. 2006;1:95-103.

17. Flensner $G$, Lindencrona $C$. The cooling-suit: case studies of its influence on fatigue among eight individuals with multiple sclerosis. J Adv Nursg. 2002;37:541-550.

18. Taradaj J. Electrical treatment of muscular spasticity. Ogólnopol Prz Med. 2002;12:15-18.

19. Miller L, Mattison P, Paul L, Wood L. The effects of transcutaneous electrical nerve stimulation (TENS) on spasticity in multiple sclerosis. Mult Scler. 2007;13:527-533.

20. Straburzyńska-Lupa A, Straburzyński G. Physiotherapy. Wydawnictwo Lekarskie PZWL, Warszawa, 2007.

21. Woldańska-Okońska M, Czernicki J. Analgesic action of magnetic fields of different characteristics. Acta Bio-Opt Inform Med. 2002;8:5-9.

22. Marz-Loose $H$, Siemes $H$. Repetitive peripheral magnetic stimulation. Treatment option for spasticity? Nervenarz. 2009;80: 1489-1495.

23. Czernicki J, Woldańska-Okońska M, Karasek M, Żylińska K, Plucińska $M$. Influence of magnetostimulation on the levels of melatonin and serotonin in serum of patients with back pain syndrome. Kwart Ortop. 2006;1:13-17.

24. Centonze D, Koch G, Versace V, et al. Repetitive transcranial magnetic stimulation of the motor cortex ameliorates spasticity in multiple sclerosis. Neurology. 2007;68:1045-1050.

25. Peszyński Drews C, Sztamska E, Klimek A. Laserotherapy in advanced progressive multiple sclerosis. Acta Bio-Optica et Informatica Medica. 2006;3:179-181. 
26. Marovino T. Cold lasers in pain management. Practical Pain Management. 2004;9:21-25.

27. Lucio AD, Perissinoto $M C$, Natalin RA, Prudente $A$, Damasceno BA Levi D'ancona CA. A comparative study of pelvic floor muscle training in women with multiple sclerosis: Its impact on lower urinary tract symptoms and quality of life. Clinics. 2011;66:1563-1568.

28. Opara J, Socha T, Prajsner A, Poświata A. Physiotherapy in stress urinary incontinence in females. Part I. Contemporary recommendations for Kegel exercises (PFME). Fizjoterapia. 2011;19:41-49.

29. Fry D, Chiara T. Pulmonary Dysfunction, Assessment, and Treatment in Multiple Sclerosis. Int J MS Care. 2010;12:97-104.

30. Schwartz I, Sajin A, Moreh E, Fisher I, Neeb M, Forest A. Robotassisted gait training in multiple sclerosis patients: $A$ randomized trial. Mult Scler. 2012;8:881-890.

31. Kargarfard M, Etemadifar M, Baker P, Mehrabi M, Hayatbakhsh R. Effect of aquatic exercise training on fatigue and health - related quality of life in patients with multiple sclerosis. Arch Phys Med Rehabil. 2012;93:1701-1708.

32. Prakash RS, Snook EM, Erickson KI, et al. Cardiorespiratory fitness: A predictor of cortical plasticity in multiple sclerosis. Neuroimage. 2007;34:1238-1244.
33. Fragoso YD, Santana DLB, Pinto RC. The positive effects of a physical activity program for multiple sclerosis patients with fatigue. Neurorehabilitation. 2008;23:153-157.

34. Dalgas U, Ingemann-Hansen T, Stenager E. Physical exercise and MS. Recommendations Int MS J. 2009;16:5-11.

35. Rietberg M, Brooks D, Uitdehaag B, Kwakkel G. Exercise therapy for multiple sclerosis. Cochrane Database Syst Rev. 2005;CD003980.

36. Wiles CM. Physiotherapy and related activities in multiple sclerosis. Mult Scler. 2008;14:863-871.

37. Rość D, Kowalik J. Quality of life of multiple sclerosis patients in relation to the degree of disability according to the Kurtzke EDSS. Zdrowie Publiczne. 2008;118:296-301.

38. Brochet B, Deloire MS, Quallet JC, Salort E, Bonnet M, Jove J, Petry KG. Pain and quality of life in the early stages after multiple sclerosis diagnosis: A 2 year longitudinal study. Clin J Pain. 2009;25:211-217.

39. Motl RW, Goldman M. Physical inactivity, neurological disability, and cardiorespiratory fitness in multiple sclerosis. Acta Neurol Scand. 2011;123:98-104.

40. Sandroff BM, Dlugnski D, Weikert M, et al. Physical activity and multiple sclerosis: New insights regarding inactivity. Acta Neurol Scand. 2012;126:256-262. 